\title{
Regionale Wirksamkeit von Hochschulen - ein Konzept zur Analyse und Bewertung
}

\author{
Rainer Vo $\beta$
}

\section{Einführung}

Der Wettbewerb zwischen Regionen bezogen auf Wirtschaftskraft, Beschäftigung und Lebensqualität wird zunehmend intensiver und die Wettbewerbsfähigkeit einer Region sowie der dort angesiedelten Unternehmen sind untrennbar miteinander verbunden. Regionen mit einem Hochschulstandort verfügen über einen strategischen Wettbewerbsvorteil. Akteure aus Wirtschaft, Verwaltung und Politik einer Region, die nicht die vielfältigen Potenziale und Kompetenzen der hier ansässigen Hochschule nutzen, verschenken einen wesentlichen Standortvorteil. Andererseits unterschätzen Hochschulen eigene Entwicklungs- und Wettbewerbschancen, wenn sie die regionalen Potenziale nicht für die Umsetzung ihrer Leistungsspektren in Lehre, Forschung und Knowhow-Transfer optimal nutzen.

Die Frage ist, wie sowohl die jeweilige Hochschule als auch die Region (Unternehmen, Verwaltungen, Verbände usw.) ihre Potenziale einsetzen und verknuipfen, um diesen Vorteil dauerhaft und entwicklungsfähig wirksam werden zu lassen. Die Art und Weise der Umsetzung dieses Vorteils - erfolgreiche Strategien, Maßnahmen und Motivationen - durch einzelne Hochschulen in ihren jeweiligen Regionen stellt eine kommunizierbare Basis für Lernprozesse dar.

In Deutschland wird die Rolle von Hochschulen für regionale Entwicklungsprozesse politisch seit Jahrzehnten betont und bewusst gefördert. In den 60er und 70er Jahren wurde in den alten Bundesländern ein flächendeckendes System von Hochschulstandorten entwickelt, das bewusst auch dezentral orientiert war, um die Entwicklung peripherer und ländlicher Regionen zu unterstuitzen. (Ganser, 1980, S. 405 f.) Bereits 1980 wurde gefordert, den wirtschaftlichen und technischen Fachhochschulen dabei eine Sonderrolle zuzuweisen und sie mit besonderen Mitteln für ihre Unterstiitzungsaktivitäten bezogen auf die regionale Wirtschaft auszustatten. (Ewers/Wettmann, 1980, S. 396)

Mit der Bildung der neuen Bundesländer nach der Wiedervereinigung Deutschlands wurden auch unter räumlichen und regionalen Gesichtspunkten Hochschulstandorte um- und neu profiliert bzw. neu errichtet und dabei ebenfalls mit dezentralen Standorten die o. g. Zielstellung verfolgt.

Die Regionalforschung in Deutschland hat sich empirisch dem Thema Hochschule und Region erstmalig vor ca. 25 Jahren und konzentriert ab Mitte der 90er Jahre zugewandt (vgl. Übersicht 1). Zum einen fällt dabei auf, dass die bisherigen Analysen im Schwerpunkt auf regionalwirtschaftliche Effekte, Wirkungen und Bedeutung abzielen, auch oft thematisiert unter „Hochschulen als Wirtschaftsfaktor", und zum anderen, dass fast ausschließlich Universitäten einbezogen und nur in knapp 17 Prozent der Fallanalysen (Münster, Berlin und Hamburg) auch Fachhochschulen berücksichtigt wurden.

Diese hier festzustellende mangelnde Repräsentanz der Fachhochschulen verwundert zumindest insofern, als der spezifische Auftrag des Gesetzgebers - durch die Verknüpfung von Wissenschaft und Praxis berufs- und anwendungsorientiert und damit besonders praxisnah auszubilden - ihre wichtige Rolle für die Wirtschaft sowie die kommunalen und sozialen Bereiche in den sie umgebenden Regionen unterstreicht. (GEW, 1996, S. 2 f.)

\begin{tabular}{|c|c|c|c|}
\hline $\mathrm{Nr}$. & Hochschule & Jahr & Autor [1] \\
\hline 1. & $\begin{array}{l}\text { Universität und } 2 \text { Fachhochschulen } \\
\text { sowie } 2 \text { Hochschulen in Münster }\end{array}$ & 1979 & Willauschus \\
\hline 2. & Universität Gießen & 1982 & Giese, u. a. \\
\hline 3. & Universität Passau & 1990 & Kleinheinz \\
\hline 4. & Universität Augsburg & 1990 & Pfaff, u. a. \\
\hline 5. & Universität St. Gallen & 1990 & Fischer/Nef \\
\hline 6. & Universität Freiburg & 1995 & Drude \\
\hline 7. & Universität Konstanz & 1995 & Oser/Schröder \\
\hline 8. & Universität Rostock & 1996 & Knödler/Tivig \\
\hline 9. & Universität Bielefeldt & 1996 & Niermann \\
\hline 10. & Technische Universität Ilmenau & 1996 & Voigt \\
\hline 11. & Universität München & 1997 & Bauer \\
\hline 12. & Universität Trier & 1997 & Benson \\
\hline 13. & $\begin{array}{l}\text { Berliner Hochschulen (4 Universitä- } \\
\text { ten, } 4 \text { Fachhochschulen) }\end{array}$ & 1997 & Geppert/Vesper \\
\hline 14. & Universität Regensburg & 1997 & Möller/Oberhofer \\
\hline 15. & $\begin{array}{l}\text { Hamburger Hochschulen } \\
\text { (3 Universitäten, } 3 \text { Hochschulen, } \\
1 \text { Fachhochschule) }\end{array}$ & 1997 & Pfähler, W. u. a. \\
\hline 15. & Universität Greifswald & 1998 & Hecht \\
\hline 16. & Universität Potsdam & 1999 & Gloede, u. a. \\
\hline 17. & $\begin{array}{l}\text { Universität Gesamthochschule- } \\
\text { Kassel }\end{array}$ & 2000 & Blume/Fromm \\
\hline 18. & Universität Halle-Wittenberg & 2001 & Schultz \\
\hline
\end{tabular}

Übersicht 1: Fallanalysen zu Wirkungen von Hochschulen auf ihre Region (nach Franz u. a. 2002; eigene Darstellung)

Viele Fachhochschulen haben sich gerade in den zurückliegenden 10 bis 15 Jahren als immer mehr an 
Bedeutung gewinnender Entwicklungsfaktor fuir ihre Regionen erwiesen, wobei ihre regionalen Wirkungen sich bei weitem nicht nur auf wirtschaftliche Aspekte beschränken. Dabei haben sie es angesichts ihrer im Vergleich zu Universitäten z. B.

- geringeren Ausstattung mit Personal,

- fehlenden wissenschaftlichen Mittelbaus,

- geringeren Mittelausstattung für Laborausrüstungen,

- wesentlich höheren Belastung der Professoren durch die Lehre und

- erschwerten Zugangs zu staatlichen Forschungsfördermitteln

einerseits nicht gerade leicht, die Aufgabenfelder Lehre in der Aus- und Weiterbildung, anwendungsorientierte Forschung sowie Dienstleistung (u. a. Technologietransfer, Beratung) auf ständig höherem Niveau zu vereinbaren und zu entwickeln. (vgl. Holtkamp, 1995) Andererseits besteht aber für jede Fachhochschule entsprechend ihres Leistungsprofils und der spezifischen Anforderungen und Entwicklungsbedingungen der Region ein breites Spektrum an Differenzierungs- und Spezialisierungsmöglichkeiten, um eine hohe regionale Wirkung in Breite und Tiefe in der Verbindung der o. g. Aufgabenfelder zu entwickeln.

Hinzu kommen neben der regionalen Dimension selbstverständlich auch nationale bzw. internationale Effekte aus den genannten Handlungsfeldern, die ihrerseits wiederum Auswirkungen auf Attraktivität und Image der Region sowie deren Hochschulen haben.

\section{Wissenstransfer als Kernprozess regio- naler Wirksamkeit von Hochschulen}

Der effiziente und zugleich effektive Transfer „fremd erzeugten“ und „selbst erzeugten“ naturwissenschaftlichen, ingenieurtechnischen, wirtschaftswissenschaftlichen, sozialwissenschaftlichen u. a. Wissens in die verschiedenen Tätigkeits- und Lebensbereiche einer Region durch eine Hochschule und deren Umsetzung in technische, wirtschaftliche und soziale Innovationen (neue bzw. verbesserte Produkte, Verfahren, Dienstleistungen, Organisationslösungen) bilden die eigentliche Basis für deren regionale Wirksamkeit (vgl. Abb. 1).

\begin{tabular}{|c|l|l|}
\cline { 2 - 3 } \multicolumn{1}{c|}{} & \multicolumn{1}{c|}{ Vorhandenes Wissen } & \multicolumn{1}{c|}{ Neu erzeugtes Wissen } \\
\hline Bildung & $\begin{array}{l}\text { Transfer zu Studenten, } \\
\text { Lehrgangsteilnehmern }\end{array}$ & $\begin{array}{l}\text { Transfer eigener FuE-Ergebnisse } \\
\text { zu Studenten }\end{array}$ \\
\hline FuE & \multicolumn{2}{|c|}{$\begin{array}{l}\text { Transfer eigener Ergebnisse zu } \\
\text { Unternehmen, Verwaltungen, } \\
\text { Politik u. a. }\end{array}$} \\
\hline Beratung & $\begin{array}{l}\text { Transfer zu Unternehmen, } \\
\text { Verwaltungen Politik u. a. }\end{array}$ & $\begin{array}{l}\text { Transfer eigener und ,fremder“ } \\
\text { FuE-Ergebnisse zu Unternehmen, } \\
\text { Verwaltungen, Politik u. a. }\end{array}$ \\
\hline & \multicolumn{2}{|c}{ explizit knowledge und/oder tacit knowledge } \\
\hline
\end{tabular}

Abb. 1: Dimensionen des Wissenstransfers aus Hochschulen

Die Adressaten des Wissenstransfers sind neben den aus- und weiterzubildenden Personen auch regionale Akteure (Organisationen und Personen) aus unterschied- lichsten gesellschaftlichen Bereichen der Region. Dabei bezieht sich Wissenstransfer immer auf zwei Wissenskategorien:

- bereits vorhandenes, so genanntes gesichertes Wissen und

- neu erzeugtes, bisher nicht vorhandenes Wissen (Wissensgenerierung).

Der Wissenstransfer aus Hochschulen erfolgt über die Bildung (i. e. S. Lehre), eigene FuE und Technologietransfer sowie die Beratungstätigkeit. Schließlich kann Wissenstransfer eine Wissenstransformation von explizitem, dokumentiertem (bekannten oder neuen) Wissen (explizit knowledge) in implizites an eine Person oder ein Team gebundenes Erfahrungswissen (tacit knowledge) und umgekehrt einschließen. Deshalb ist Wissenstransfer oft an „face to face-Kontakte“ oder sogar an Personaltransfer (Wissensträger) gebunden.

Die regionale Wirksamkeit von Hochschulen basiert demnach auf einem System des Wissensmanagement, das Wissensgenerierung, Wissenstransformation und Wissenstransfer in seiner wechselseitigen Bedingtheit und Durchdringung umfasst. Hier geht es bei weitem nicht nur um Technologietransfer oder Know-how, sondern die Akteure der Hochschule müssen in vernetztem Zusammenwirken mit regionalen Akteuren, z. B. aus Unternehmen, anderen Wissenschaftseinrichtungen, Technologie- und Gründerzentren sowie Verwaltungen, diesen komplexen Zusammenhang aufeinander bezogen aktiv gestalten (vgl. Abb. 2).

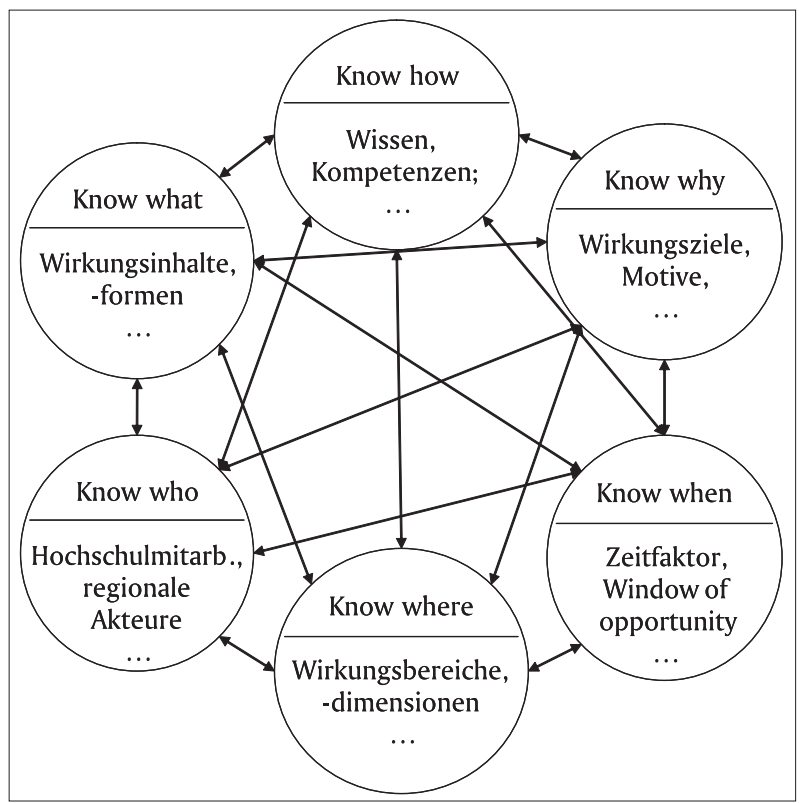

Abb. 2: Wissensmanagement für die regionale Wirksamkeit der Hochschulen

Beispielsweise gibt es entsprechend der Akzentuierung ihres gesellschaftlichen Auftrags sowohl Unterschiede hinsichtlich des „Know why“ zwischen Universitäten und Fachhochschulen als auch zwischen den jeweiligen Hochschulen und ihren regionalen Partnern in Wirtschaft, Verwaltung und Politik. Dies hat sicher auch Konsequenzen für Erwartungen und Angebote bezogen auf das „Know how“ und „Know what“ aber auch des „Know who“ für die an der Leistungserbringung bzw. 
der Schaffung der dafür erforderlichen regionalen Voraussetzungen zu beteiligenden Akteure innerhalb und außerhalb der Hochschule. Schließlich ist auch Konsens iiber Anspruch und Machbarkeit bezogen auf das „Know where" zwischen den Beteiligten zu erzielen und das „Know when“ nicht zu unterschätzen. Es können auch gut gemeinte Wirkungsabsichten von Hochschulen auf die Region zu früh oder zu spät initiiert werden bzw. für bestimmte Wirkungsbereiche gibt es ein ,window of opportunity“. Vor zehn Jahren beispielsweise wurde ein solches „Fenster“ gerade geöffnet, als die Stadt Luckenwalde und der Landkreis Teltow-Fläming begannen, mit Unterstuitzung der Universität Potsdam, der Technischen Universität Berlin und der Technischen Fachhochschule Wildau, den heute erfolgreichen Biotechnologiepark in Luckenwalde „auf der grünen Wiese“ aufzubauen. (Große u. a., 1998) Vergleichsweise Ähnliches in diesem Technologie- und Wirtschaftsbereich wäre heute aus verschiedenen Gründen nicht mehr machbar, dieses Fenster hat sich geschlossen.

Zum damaligen Zeitpunkt hätte die Technische Fachhochschule Wildau aber auch keinen regionalen Wirkungsbereich „Luft- und Raumfahrttechnik“ anzielen und mitgestalten können, weder vom Entwicklungsstand der Region, noch von den Interessen der Akteure in Politik und Wirtschaft oder den Kompetenzen und der Leistungskraft der Fachhochschule her betrachtet. Dieses „Fenster“ wird gerade von den interessierten Akteuren weiter „aufgestoßen“. Beide „Fenster“ müssen damals wie heute beständig „geölt" und auch bei „Gegenwind offen gehalten und weiter aufgedrückt" werden, womit man wieder beim geeigneten „Know who“, „Know what" usw., also dem vernetzten Wissensmanagement für die Entwicklung und Erschließung der regionalen Wirkungspotenziale von Hochschulen, angelangt ist.

\section{Regionale Wirkungsräume von Hochschulen}

\subsection{Der generelle Wirkungsraum}

Dieses Verständnis von Wissenstransfer und Wissensmanagement eröffnet den Zugang zu unterschiedlich inhaltlich orientierten und dimensionierten regionalen Wirkungsräumen einer Hochschule.

Die Beantwortung der Fragen

1. Welche Funktionen kann eine Hochschule erfüllen, was sind ihre Leistungsangebote? (x-Achse)

2. Wie, mit welchen Methoden kann sie diese hervorbringen? (y-Achse)

3. Wer benötigt, nutzt solche Funktionen und Leistungsangebote? (z-Achse)

führt dadurch, dass auf jede Frage mehrere alternative Antworten möglich sind, zu einem generellen idealtypischen Handlungs- und Wirkungsraum für Hochschulen (vgl. Abb. 3). In diesen Raum kann man nun verschiedene Schnitte legen und durch Kombination ausgewählter Parameter Teilräume bilden, die unterschiedliche Schwer-

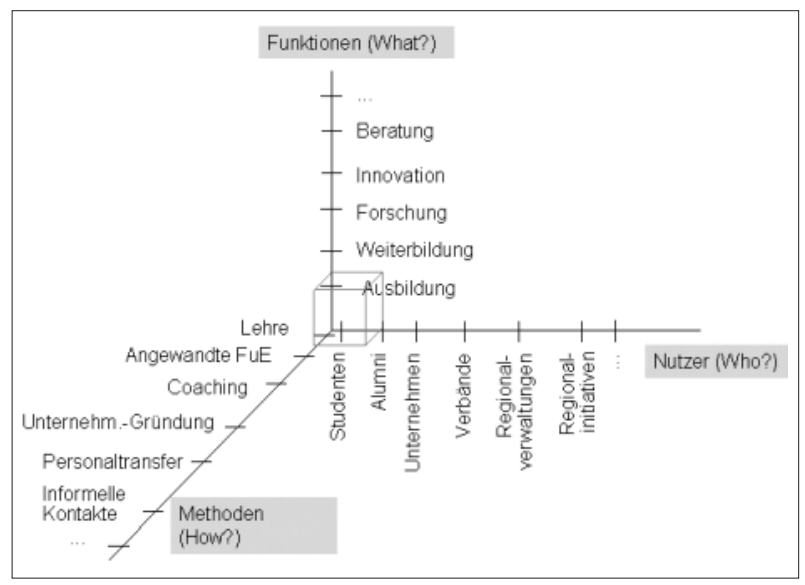

Abb. 3: Genereller regionaler Wirkungsraum von Hochschulen

punkte einzelner Hochschulen beschreiben. Der Teilraum „Bildung durch Lehre“ wird sicher als Minimum von jeder Hochschule ausgefuillt.

\subsection{Regionaler Wirkungsraum „Bildung“}

Innerhalb des Teilraums „Bildung“ sind verschiedene Spezialisierungs- und Differenzierungsmuster möglich (vgl. Abb. 4). Eine Hochschule bietet vielleicht nur das Direktstudium an, eine andere auch die Möglichkeit des Fernstudiums, eine weitere Hochschule hat duale Studiengänge, eine andere nicht. Die eine Hochschule hat z. B. neben Studiengängen der Wirtschaft und Wirtschaftsinformatik auch mehrere ingenieurtechnische Studiengänge und damit auch den Status einer Technischen Universität oder Technischen Fachhochschule, eine andere nicht usw.

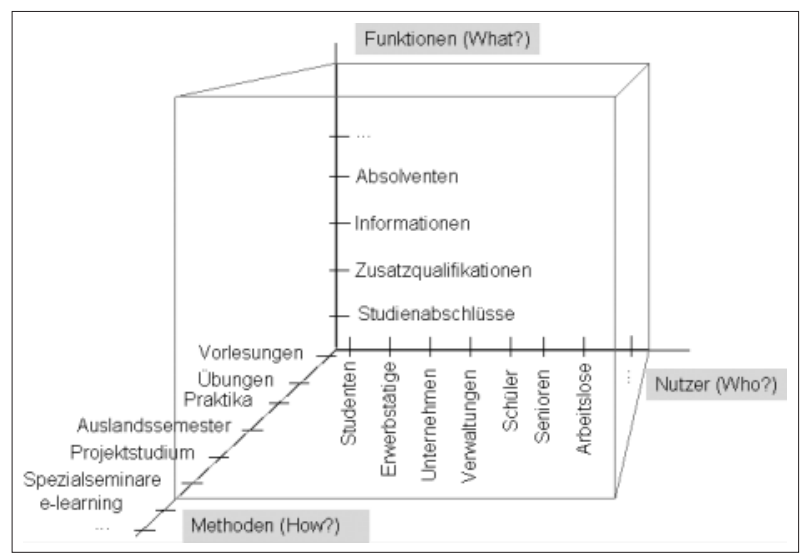

Abb. 4: Regionaler Wirkungsraum „Bildung“ von Hochschulen

Im Folgenden sollen nun immer wieder die Erfahrungen der Technischen Fachhochschule Wildau (TFHW) eingefügt werden, um das Konzept der Analyse und Bewertung der regionalen Wirksamkeit von (Fach-)Hochschulen zu illustrieren. [2]

Die TFHW zählt unter den fünf Fachhochschulen des Landes Brandenburg mit 3.200 Studierenden im Jahre 2003 zu den zwei größten. Sie wurde im Jahre 1991 gegründet, ist gewissermaßen aus der traditionsreichen Ingenieurschule für Maschinenbau Wildau hervorgegangen und liegt unmittelbar an der südlichen Stadtgrenze der Metropole Berlin, noch im Bereich 
des Schnellbahnnetzes der Hauptstadt. Mit ihren drei Fachbereichen „Ingenieurwesen/Wirtschaftsingenieurwesen“, „Betriebswirtschaft/Wirtschaftsinformatik“ und „Wirtschaft, Verwaltung und Recht", die 14 Direktstudiengänge und sechs Fernstudiengänge anbieten und dabei alle in der Abbildung 3 aufgefuihrten Lehrformen und Bildungsmaßnahmen anwenden, deckt die TFHW den dargestellten Wirkungsraum „Bildung“ ab. Spezialseminare für unterschiedliche Zielgruppen aus Wirtschaft und Verwaltung oder für Senioren sind üblich.

Standortgunst und moderne Studienangebote tragen zur Attraktivität der TFH Wildau bei, die wie von einer Fachhochschule zu erwarten, hinsichtlich der Herkunft ihrer Studierenden stark regional verortet ist. So kommen etwa 71 Prozent aus der Region (50 Prozent aus dem Land Brandenburg und 21 Prozent aus Berlin), 13 Prozent aus weiteren neuen und zehn Prozent aus den alten Bundesländern sowie sechs Prozent aus dem Ausland (von Polen über die Niederlande bis Guatemala). Studentenaustausch besteht mit vielen Ländern, neben mittel- und osteuropäischen Staaten z. B. auch mit Dänemark, Finnland, den Niederlanden und Irland.

Ausdruck der Attraktivität der TFH Wildau ist auch die ungebrochen hohe und teilweise wachsende Nachfrage nach Studienplätzen. So kamen beispielsweise im Immatrikulationsjahr 2003/04 auf einen Studienplatz in der Betriebswirtschaftslehre etwa sechs Bewerber, bei den Wirtschaftsingenieuren fünf und in der Logistik, Wirtschaftsinformatik, Wirtschaft und Recht, Verwaltung und Recht mehr als vier. Selbst in den technischen Studiengängen bewarben sich drei Studienberechtigte auf einen Platz. Hier war es noch im Jahr 1998/99 nur ein Bewerber pro Studienplatz. Die Folge dieser hohen Nachfrage ist, dass die TFH Wildau als einzige Fachhochschule im Land Brandenburg auf alle 14 Direktstudiengänge den numerus clausus als Zugangsbeschränkung hat. Dies wirkt sich wiederum positiv auf die Quote von „Studienabbrechern“ und auf die Zahl und Qualität der Absolventen aus. Von der wachsenden Zahl der Absolventen finden etwa 50 Prozent eine Beschäftigung in der Region (21 Prozent im Land Brandenburg und 29 Prozent in Berlin), sieben Prozent in den anderen neuen und 34 Prozent in den alten Bundesländern, während etwa sechs Prozent eine Beschäftigung im Ausland aufnehmen. [3] Auch diese Zahlen sind Ausdruck der regionalen Wirksamkeit der TFH Wildau und ihres überregional guten Images.

Woanders oder zukünftig wird es vermutlich je nach Nachfrage und Angebot in verschiedenen Regionen noch weitere Wirkungsmöglichkeiten im Handlungsraum „Bildung" fuir Hochschulen geben.

\subsection{Regionaler Wirkungsraum „Forschung und Entwicklung“"}

Ein weiterer für den Wissenstransfer wichtiger Wirkungsraum ergibt sich aus den Aktivitäten einer Hochschule in der angewandten Forschung und Entwicklung (vgl. Abb. 5).

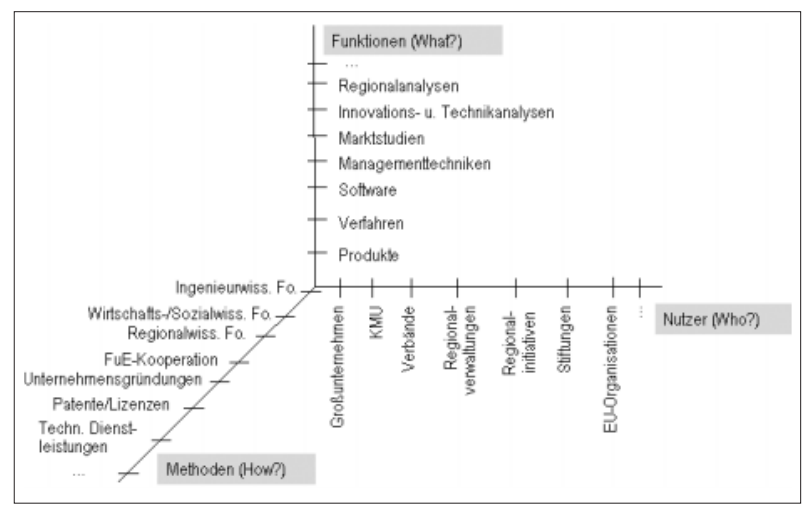

Abb. 5: Regionaler Wirkungsraum „Forschung und Entwicklung“ von Hochschulen

Hier wird auch die inhaltliche Verknüpfung unterschiedlicher Dimensionen möglicher regionaler Wirkungen deutlich. So kann z. B. eine Hochschule nur dann technisch-technologisches Wissen in die Unternehmen der Region transferieren und dabei Forschungskooperationen realisieren, wenn sie in ihrer Grundfunktion „Bildung“ ingenieurtechnische Studiengänge anbietet und uiber entsprechende Fachleute verfuigt. Universitäten sind in ihren Forschungsprojekten mehr auf die Grundlagenforschung orientiert, während Fachhochschulen stärker anwendungsbezogen FuE betreiben.

Es verwundert sicher nicht, dass die TFH Wildau bei ihren diversifizierten Studienangeboten diese Synergien nutzt und auch über entsprechende Potenziale angewandter Forschung und Entwicklung von ingenieurtechnischen Disziplinen bis hin zu sozial- und kommunalwissenschaftlicher Forschung verfuigt und damit den dargestellten Wirkungsraum komplett bedient. Sie zählt unter den ca. 150 Fachhochschulen in Deutschland mit zu den führenden in der Einwerbung von Drittmitteln durch angewandte Forschung und Projekte für öffentliche Auftraggeber bzw. die Wirtschaft.

Die TFH Wildau hat ca. 170 Beschäftigte plus etwa 60 zusätzliche Drittmittelbeschäftigte. Damit gleicht sie einen Hauptnachteil von Fachhochschulen - das Fehlen eines Haushalt finanzierten wissenschaftlichen Mittelbaus - teilweise zu Gunsten verstärkter Wirksamkeit aus Forschung und Entwicklung aus. Die TFH Wildau konnte ihre Einnahmen aus Drittmitteln in den zurüickliegenden fünf Jahren mehr als verdoppeln auf eine Summe von 3,3 Mio. $€$ im Jahre 2003. Das bedeutet, bezogen auf die Summe des Haushaltes der Hochschule im Jahre 2003 von etwa 9 Mio. €, zusätzlich 33 Prozent an Finanzmitteln, die für die Wirksamkeit der Fachhochschule eingesetzt werden konnten. Diese Leistung gewinnt noch an Gewicht, wenn man berücksichtigt, dass in den alten Bundesländern von allen Hochschulen (einschließlich der Universitäten) bezogen auf ihre Haushaltsmittelsumme zusätzlich 11 Prozent Drittmittel eingeworben werden und für alle neuen Bundesländer dieser Anteil etwa sieben Prozent ausmacht. (Franz u. a. 2001, S. 33 f.)

Die Hinzuziehung eines weiteren Indikators macht spätestens an dieser Stelle deutlich, dass regionale Wirkungen von Hochschulen auch von den jeweiligen regionalen 
Entwicklungsbedingungen, den Wirtschaftsstrukturen, von der Nachfrage nach bestimmten Leistungsangeboten, vom Vorhandensein und der Leistungsfähigkeit potenzieller Anwender abhängen. Betrachtet man den Anteil an den Drittmitteln aus der gewerblichen Wirtschaft, dann machte dieser im Jahre 1999 für die alten Bundesländer mehr als 30 Prozent, für die neuen Länder ca. 24 Prozent und fuir das Land Brandenburg 25 Prozent aus. (Rosenfeld/ Heimpold 2001, S. 398, eigene Berechnungen)

Der Anteil von Drittmitteln aus der Wirtschaft der TFH Wildau insgesamt betrug im Jahre 2003 „erst“ 16 Prozent. Dies verweist sowohl auf Reserven der Hochschule in der Akquise als auch auf die Nachfrageschwäche der überwiegend kleinen Unternehmen in der Region, was darin zum Ausdruck kommt, dass nur 41 Prozent der wirtschaftsbezogenen Drittmittelsumme aus Unternehmen des Landes Brandenburg stammen. Es fehlen Großunternehmen in der Region, die in dieser Beziehung eine „Leuchtturmfunktion“ übernehmen könnten. Hinzu kommt, dass es in den neuen Bundesländern, verglichen mit den alten, bezogen auf die Einwohnerzahl immer noch 100000 Unternehmen zu wenig gibt. (Heimpold 2003, S. 330) Diese Situation spiegelte sich auch schon bei den o. g. Einsatzregionen der Absolventen wieder, indem mehr als die Hälfte von ihnen außerhalb der Region Berlin/Brandenburg beruflich tätig sind.

Eine bemerkenswerte Erfahrung der TFH Wildau besteht darin, dass es inzwischen stabil gelingt, jährlich 10 bis 12 Prozent der Drittmittel mit Projekten aus dem Fachbereich Betriebswirtschaft/Wirtschaftsinformatik einzuwerben. Dadurch wird das regionale Wirkungsspektrum sowohl inhaltlich als auch hinsichtlich der regionalen Akteure als Nutzer wesentlich verbreitert. Die TFH Wildau forscht inzwischen auf mehr als 20 Gebieten. Mindestens sechs davon sind wirtschafts-, sozial- und regionalwissenschaftliche Forschungsschwerpunkte (vgl. Übersicht 2).

\begin{tabular}{|l|l|}
\hline CAD/CAM-Lösungen, Fertigung & Marketing \\
\hline Computergestützte Organisation & Materialforschung \\
\hline $\begin{array}{l}\text { Energieverfahrenstechnik/ } \\
\text { Erneuerbare Energien }\end{array}$ & Mess- und Sensortechnik \\
\hline Facility-Management & Oberflächentechnik \\
\hline Gründungsforschung & Qualitätsmanagement \\
\hline Kreislaufwirtschaft/Recycling & Regionale Innovationsforschung \\
\hline Kunststofftechnik & Technikfolgenabschätzung \\
\hline Laser- und Plasmatechnik & Telekommunikation \\
\hline Logistik & Umwelttechnologien \\
\hline Managementtechniken & $\begin{array}{l}\text { Verfahrenstechnik/ } \\
\text { Abwasseraufbereitung }\end{array}$ \\
\hline
\end{tabular}

Übersicht 2: Schwerpunkte in der angewandten Forschung und Entwicklung an der Technischen Fachhochschule Wildau

Eine zunehmend an Bedeutung gewinnende Plattform zur Hervorbringung und Umsetzung neuen Wissens ist die Gründung von Unternehmen durch oder unter Beteiligung von Studenten und Mitarbeitern der Hochschulen. Die TFH Wildau ist hinsichtlich des Gründungsklimas für solche spin offs, ausgedruickt in der Zahl entwickelter
Businesspläne je Student und der tatsächlich gegründeten Unternehmen, fuihrend in der Region und wurde in den letzten Jahren zweifacher Träger der Auszeichnung „Beste Ideenschmiede des Landes Brandenburg“.

\subsection{Regionaler Wirkungsraum „Beratung“}

Schließlich ergibt sich neben der Bildung und der angewandten Forschung und Entwicklung noch ein dritter regionaler Wirkungsraum für den Wissenstransfer in die Praxis, der sich beständig ausweitet und umstrukturiert (vgl. Abb. 6).

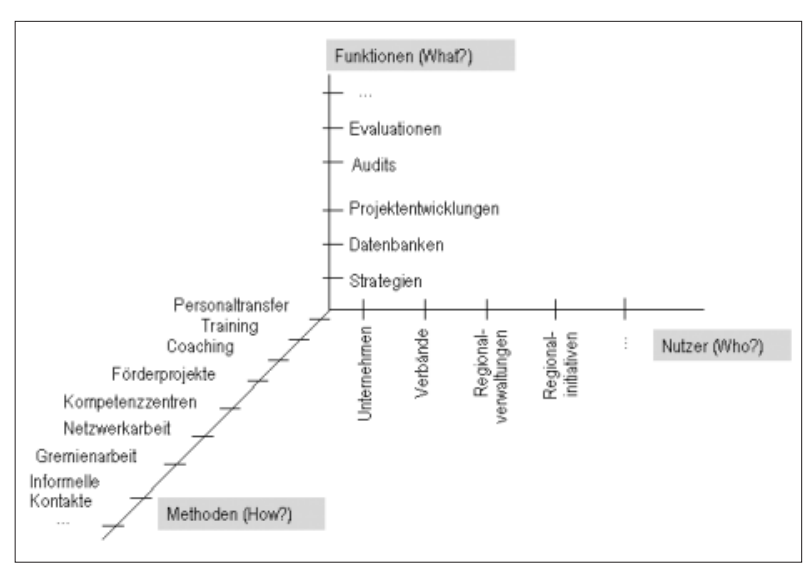

Abb. 6: Regionaler Wirkungsraum „Beratung“ von Hochschulen

Auch hier werden Hochschulen nach ihren Profilen und Potenzialen unterschiedliche Teilräume auswählen und verschiedene Nutzergruppen erreichen. Die Schnittflächen dieses Wirkungsraums bezogen auf Stärken und Schwächen einer Hochschule in den beiden zuvor betrachteten Wirkungsräumen sind kaum zu überschätzen. Breite und differenzierte Leistungsangebote in Lehre und Forschung ermöglichen die vielfältigsten Wirkungen durch beratende Tätigkeiten mit unterschiedlichsten Methoden für verschiedene regionale Akteursgruppen. An diesem Wirkungsraum wird deutlich, dass es auch fuir die regionalen Wirkungen so genannte „harte“ (direkt messbare) und „,weiche“ (indirekt messbare, vom persönlichen Kontakt und Engagement getragene) Wirkungsbereiche und -formen gibt. Die regionale Wirksamkeit von Hochschulen reduziert sich bei weitem nicht auf die regionalwirtschaftlichen Effekte. Es ist ähnlich wie bei der Rolle von harten oder weichen Standortfaktoren für Unternehmen oder für die Attraktivität einer Stadt. Man kann sie nicht gegeneinander aufwiegen, sie stehen in nur schwer durchdringbaren Verknüpfungen und Wechselwirkungen.

Das, was in und über diesen Raum an regionalen Wirkungen erzielt werden kann, wird bei guter Ausprägung und Qualität entsprechender Aktivitäten meist von allen Beteiligten innerhalb und außerhalb der Hochschule als die eigentlich dauerhafte Basis, als Voraussetzung und Umsetzungsgrundlage vieler anderer regionaler Wirkungen hochgeschätzt und entsprechend ,gepflegt“.

Wachsende Bedeutung kommt hier der Mitwirkung der Hochschule an sowie der wissenschaftlichen Begleitung bzw. Evaluation von Strategieentwicklungsprozessen 
bei Unternehmen, Verbänden und Regionalverwaltungen zu. Fachleute der TFH Wildau sind in dieser Beziehung in viele Projekte und Aktivitäten involviert: Dies insbesondere uiber die Mitarbeit in verschiedenen Gremien wie Strategiekreise, Arbeitsgruppen (z. B. zur Erarbeitung regionaler Entwicklungskonzepte) und über die Netzwerkarbeit. Die Fachhochschule ist an mehreren Innovations- und Bildungsnetzwerken beteiligt bzw. hat solche selbst initiiert. Sie entwickelt mehrere naturwissenschaftlich-technische Kompetenzzentren (z. B. Lasertechnologie, Luft- und Raumfahrt, Logistik, Telematik) aber auch wirtschafts- und sozialwissenschaftliche Kompetenzknoten (Innovations- und Regionalmanagement, Gründungsmanagement) unter Beteiligung von und mit Orientierung auf die in der Abbildung 6 genannten regionalen Nutzergruppen.

\section{Wechselwirkung von Hochschule und Region}

Es muss immer wieder betont werden, dass eine breite und spürbar positive regionale Wirksamkeit neben der Kompetenz und Motivation der Professoren, der Hochschulleitung und aller Mitarbeiter wie auch der Studenten bestimmte regionale Bedingungen zur Voraussetzung hat. Wenn eine Region solche Effekte aus den Tätigkeitsbereichen ihrer Hochschule haben und nutzen will, müssen auch dafuir erforderliche regionale Voraussetzungen gegeben sein bzw. entwickelt werden.

Die Leistungsfähigkeit einer Hochschule, ihre Wirkungen und die regionalen Entwicklungsbedingungen hängen wechselseitig zusammen. Der Grundzusammenhang ist in Abbildung 7 dargestellt.

\begin{tabular}{|c|c|}
\hline $\begin{array}{l}\text { Fachhochschulen haben } \\
\text { bezogen auf die Region ein }\end{array}$ & $\begin{array}{l}\text { Regionen haben bezogen } \\
\text { auf Fachhochschulen ein }\end{array}$ \\
\hline Angebotspotential - AP (H) & Versorgungspotential - VP (R) \\
\hline $\begin{array}{l}\text { - aus- und weitergebildete Personen } \\
\text { - Forschungsergebnisse } \\
\text { - Beratungsleistungen } \\
\text { - Unternehmensgründungen } \\
\text {-... }\end{array}$ & $\begin{array}{l}\text {-Altersstruktur, Bildungsstruktur } \\
\text {-Infrastrukturen } \\
\text { - Arbeitsmarkt } \\
\text { - Freizeitangebote } \\
\text { - Umweltqualität } \\
\text {-... }\end{array}$ \\
\hline Beanspruchungspotential - BP $(\mathrm{H})$ & Nachfragepotential - NP (R) \\
\hline $\begin{array}{l}\text { - Flächen und Immobilien } \\
\text {-Arbeitskräfte } \\
\text { •Infrastrukturen } \\
\text { •... }\end{array}$ & $\begin{array}{l}\text { - Ausgebildetes Personal } \\
\text { - Innovationen } \\
\text { - Beratungsleistungen } \\
\text {-... }\end{array}$ \\
\hline $\begin{array}{l}\text { 1) } A P(H) \quad \sum N P(R) \\
\text { 2) } A P(H)=f[V P(R): B P(H)] \\
\text { 3) } B P(H) \quad \geqq V P(R) \\
\text { 4) } B P(H)=f[N P(R): A P(H)]\end{array}$ & \\
\hline
\end{tabular}

Abb. 7: Grundzusammenhang von Hochschulen (H) und Region (R)

Das Angebotspotenzial einer Hochschule und das auf ihr Leistungsspektrum bezogene und aus den Entwicklungszielen und -erfordernissen abgeleitete Nachfragepotenzial der Region können sich auf einem höheren, wettbewerbsfähigen oder auf einem niedrigeren, entwicklungsbedürf- tigen Niveau entsprechen oder aus der Sicht regionaler Wirkungen im Sinne von größer oder kleiner voneinander abweichen (vgl. Beziehung 1 in Abb. 7).

Wenn die Hochschule durch Wissenstransfer fördernd zur Regionalentwicklung beitragen, also ihr Angebotspotenzial wirksam machen soll, muss sie in der Region entsprechende Bedingungen vorfinden. Die Region muss den Anspruch, den Hochschulen an regionale Bedingungen stellen, durch eine entsprechende Versorgungsleistung befriedigen (vgl. Beziehung 2 in Abb. 7). Dabei können sich das Beanspruchungspotenzial der Hochschule und das Versorgungspotenzial auf einem mehr oder weniger wünschenswerten Niveau entsprechen oder aber voneinander in verschiedener Richtung abweichen (vgl. Beziehung 3 in Abb. 7). Schließlich ist das Beanspruchungspotenzial einer Hochschule nach Inhalt und Intensität immer abhängig davon, auf welchem Niveau sich das Verhältnis von Nachfragepotenzial der Region und Angebotspotenzial der Hochschule bewegt (vgl. Beziehung 4 in Abb. 7). Aus solchen Wechselbeziehungen ergeben sich Spannungsfelder, die sich stets auf neuem Niveau reproduzieren - bedingt durch die Entwicklungen in der Hochschule und der Region. Solche Spannungsfelder lassen sich mit der Portfolioanalyse beschreiben, aus der bestimmte Handlungsstrategien der Hochschule wie auch der Region abgeleitet werden können, die dann zu einer auf hohem Niveau abgestimmten Entwicklung ihrer jeweiligen Potenziale führen (vgl. Abb. 8).

\begin{tabular}{|c|c|c|}
\hline \multirow{2}{*}{ 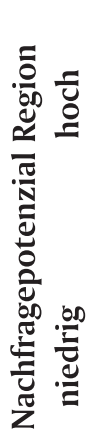 } & $\begin{array}{l}\text { Region muss ihr } \\
\text { Versorgungspotenzial } \\
\text { fuir H stärken, } \\
\mathrm{H} \text { muss sich } \\
\text { profilieren }\end{array}$ & $\begin{array}{l}\text { Beide Seiten sind } \\
\text { Gewinner }\end{array}$ \\
\hline & $\begin{array}{l}\text { Beide Seiten müssen } \\
\text { sich stärker } \\
\text { wahrnehmen und } \\
\text { einbinden }\end{array}$ & $\begin{array}{l}\text { Region muss } \\
\text { Angebotspotenzial } \\
\text { der H besser } \\
\text { nutzen }\end{array}$ \\
\hline & $\begin{array}{l}\text { niedrig } \\
\text { Angebotspoten }\end{array}$ & $\begin{array}{r}\text { hoch } \\
\text { zial Hochschule }\end{array}$ \\
\hline
\end{tabular}

Abb. 8: Portfolio - Regionale Wirksamkeit von Hochschulen (H)

Wenn beide - Region und Hochschule - beständig um höhere Wettbewerbsfähigkeit bemüht sind und sich aufeinander bezogen und miteinander entwickeln, sollte sich das Spektrum der regionalen Wirkungen der Hochschule in den zuvor diskutierten Handlungsräumen zunehmend verbreitern und vertiefen.

Der Versuch, die TFH Wildau in einem solchen Portfolio zu positionieren, lässt vielleicht nach den vorausgehend angefuihrten Beispielen für die bisherigen regionalen Wirkungen die dargestellte Verortung begründet erscheinen. Die verschiedenen regionalen Akteursgruppen nutzen die Leistungen der TFH Wildau jedoch noch nicht im möglichen Maße. Die Angebote der Technischen Fachhochschule werden sich immer wieder an zu erwarteten Nachfrageentwicklungen der Region profilieren müssen. 


\begin{tabular}{|c|c|c|}
\hline $\begin{array}{l}\text { Regionale Wirkungen von Hochschulen } \\
\text { (Beispiele) }\end{array}$ & Regionale Entwicklungsbedingungen & Voraussetzungen für Hochschulen (Beispiele) \\
\hline Zuwanderung junger Menschen zum Studium & demographische Struktur & ausreichende Zahl junger Menschen \\
\hline $\begin{array}{l}\text { Hochschule als wichtiges Element der Bildungs- } \\
\text { infrastruktur }\end{array}$ & Bildungsinfrastruktur & Schulen mit Abschlüissen der Hochschulreife \\
\hline $\begin{array}{l}\text { Hochschule als Schlüsselakteur in Regionalen } \\
\text { Innovationssystemen }\end{array}$ & Forschungsinfrastruktur & $\begin{array}{l}\text { Vorhandensein von kooperierenden Forschungs- } \\
\text { einrichtungen }\end{array}$ \\
\hline $\begin{array}{l}\text { Zusammenarbeit mit Unternehmen; } \\
\text { Unternehmensgründungen aus der Hochschule }\end{array}$ & Wirtschaftsstruktur & $\begin{array}{l}\text { Vorhandensein moderner Branchen, großer und } \\
\text { mittelständischer Unternehmen }\end{array}$ \\
\hline $\begin{array}{l}\text { Bereitstellung qualifizierter Absolventen; } \\
\text { Wissenstransfer in Unternehmen }\end{array}$ & Wirtschaftskraft & $\begin{array}{l}\text { entwicklungsfähige, technologie-orientierte } \\
\text { Unternehmen verschiedener Branchen }\end{array}$ \\
\hline $\begin{array}{l}\text { Schaffung von Arbeitsplätzen in Hochschulen; } \\
\text { Weiterbildung von Arbeitslosen }\end{array}$ & Arbeitsmarkt & $\begin{array}{l}\text { qualifiziertes technisches und kaufmännisches } \\
\text { Personal }\end{array}$ \\
\hline $\begin{array}{l}\text { technisch-technologische Beiträge zur } \\
\text { Modernisierung der Infrastruktur }\end{array}$ & technische Infrastruktur & Leistungsfähige Ver- und Entsorgungssysteme \\
\hline $\begin{array}{l}\text { Hochschule unterstiitzt Umwelt- und Natur- } \\
\text { schutz mit Know-How }\end{array}$ & Natur und Umwelt & gute Qualität als Standortfaktor \\
\hline $\begin{array}{l}\text { Hochschule repräsentiert Region nach außen } \\
\text { und beteiligt sich an Netzwerken }\end{array}$ & externe regionale Verflechtungen & $\begin{array}{l}\text { Förderung von überregionalen Kontakten zu anderen } \\
\text { Fachhochschulen und Unternehmen }\end{array}$ \\
\hline $\begin{array}{l}\text { Hochschule unterstuitzt Kommunen und } \\
\text { Behörden bei der Verwaltungsreform }\end{array}$ & Politisch-administrative Struktur & $\begin{array}{l}\text { Verwaltungen und Parteien fördern Entwicklung der } \\
\text { Hochschule }\end{array}$ \\
\hline
\end{tabular}

Übersicht 3: Regionale Entwicklungsbedingungen als Voraussetzung und Ziel von Wirkung von Hochschulen

Dieser Zusammenhang, in dem regionale Entwicklungsbedingungen Wirkungsziel und Wirkungsvoraussetzung für Aktivitäten von Hochschulen in den vorher dargestellten Wirkungsräumen sind, ist in der Übersicht 3 beispielhaft illustriert. Hier wird noch einmal deutlich, dass sich regionale Wirkungen von Hochschulen bei weitem nicht auf regionalwirtschaftliche bzw. ökonomische Wirkungen reduzieren lassen.

\section{Verständnis von Region als Wirkungs- raum von Hochschulen}

Der räumliche Kontext, in dem regionale Wirkungen von Hochschulen angezielt und verortet sind sowie analysiert und bewertet werden können, kann bezogen auf ein und dieselbe Hochschule durchaus verschieden zweckdefiniert und dimensioniert sein.

Regionen sind zunächst territorial unterschiedlich dimensionierte Lebensräume, die über komplexe ökologische, wirtschaftliche, soziale und politische Systeme definierbar sind. Sie verfügen über eine kritische Masse leistungsfähiger endogener Potenziale (regionaler Entwicklungsbedingungen) und exogener Verflechtungsbeziehungen. Diese gestatten es den hier arbeitenden und lebenden Menschen mit ihren spezifischen Kenntnissen, Fähigkeiten und Fertigkeiten, Produkte und Dienstleistungen hervorzubringen und zu vermarkten sowie darüber eine hohe Lebensqualität und zukunftsfähige Regionalentwicklung zu sichern. (Voß, 2001, S. 28 f.) Zugleich sind sie offene funktionale Beziehungsgefuige und Interessenfelder von privaten, öffentlichen und gemeinnuitzigen regionalen Akteuren aus Wirtschaft, Wissenschaft, Bildung, Politik und weiteren institutionalisierten sowie nicht institutionalisierten Gruppen.

Ein solches Verständnis von Region überwindet politische und raumplanerische Grenzen und öffnet die Sicht auf Regionen, die sich aus Netzwerken räumlicher
Akteure konstituieren. Solche Netzwerke bilden sich oft als Träger von raumübergreifenden Projekten, z. B. in den Bereichen Forschung und Innovation, Industrie, Landwirtschaft und Landschaftsgestaltung sowie soziale Entwicklungsinitiativen heraus, deren Ziele, Ressourcen und Wirkungen eine „neue, andere Region“ definieren. (vgl. Kuhn, 2002) Somit kann das räumliche Bezugssystem für regionale Wirkungen einer Hochschule „sowohl als auch“ bzw. „und/oder" sein:

- die Stadt(region), in der die Hochschule ihren Standort hat;

- der entsprechende Landkreis;

- der Teilraum eines Bundeslandes (z. B. Südbrandenburg);

- das Bundesland;

- über Projekte und Akteursbeziehungen konstituierte Regionen.

Allerdings wird ausgehend von den unterschiedlich akzentuierten gesellschaftlichen Aufträgen für Universitäten bzw. Fachhochschulen und auf der Basis langjähriger Erfahrungen aus den alten Bundesländern, gestuitzt auf empirische Erhebungen, darauf verwiesen, „dass gerade Universitäten häufig nur gering mit ihrem regionalen Umfeld verflochten sind und interregionale/internationale Verflechtungen überwiegen. (...) Sie erfüllen eine Radarfunktion für die regionale Wirtschaft und stellen im Sinne eines Brïckenkopfes die Verbindung zu internationalen Wissenschafts- und Technologienetzwerken her.“ (Koschatzky/Zenker 1999, S. 5 f.)

Bezogen auf die regionale Verortung von Fachhochschulen wird die Situation anders gesehen. „Sie bedienen meist eine regionale Firmenklientel, auf deren Branchen- und Technologieprofil sie zugeschnitten sind. Räumlich nahe Kooperationsbeziehungen überwiegen. Da betriebliche Aspekte in der Fachschulausbildung eine größere Rolle spielen als im Universitätsstudium, sind die Kontaktbarrieren zwischen den Unternehmen einer Region und ihrer Fachhochschule niedriger als bei 
Universitäten, da beide aufeinander angewiesen sind. In den neuen Bundesländern ist eine stärkere Neigung zu beobachten, auf Forschungseinrichtungen aus der Region oder aus Ostdeutschland generell zurückzugreifen als in Westdeutschland.“ (Koschatzky/Zenker 1999, S. 6)

Die Notwendigkeit eines solchen andersgearteten Kooperationsverhaltens ist angesichts der Tatsache, dass in Ostdeutschland die FuE-Intensität in der Wirtschaft, trotz positiver Zuwächse in den letzten Jahren (vgl. FuE Info 2003), um ein Mehrfaches geringer ist als in Westdeutschland und FuE-intensive, international agierende Großunternehmen als bevorzugte Partner von Universitäten und wichtige Wirtschaftspartner von KMU in den neuen Bundesländern kaum vorhanden sind, nur zu unterstreichen. Die Hochschulen können in Ostdeutschland diese traditionelle Arbeitsteilung zwischen Fachhochschulen und Universitäten hinsichtlich ihrer regionalen Orientierung und Wirkungen nicht in gleicher Weise ausprägen bzw. aufrechterhalten.

Bei entsprechenden regionalen und institutionellen Voraussetzungen sowie persönlichen Kompetenzen, Motivationen und aktivem Kooperationsverhalten können Mitarbeiter von Hochschulen nachhaltige regionale Wirkungen in unterschiedlicher räumlicher Kontextualisierung erzielen.

Auf diese Weise wird die TFH Wildau durch ihr Zusammenwirken mit Unternehmen, Kammern und Verbänden, anderen Forschungs- und Bildungseinrichtungen, Landkreis- und Kommunalverwaltungen usw. zunehmend zu einem der Knoten im Netz der Bildungs-, Forschungs- und Transfereinrichtungen des Landes Brandenburg. Allein die Aktivitäten in den Wirkungsräumen Forschung, Beratung und Bildung, die vom Autor dieses Beitrages und von seinen Mitarbeitern getragen werden, bilden ein immer dichter werdendes kleines Netz über diese Region (vgl. Abb. 9).

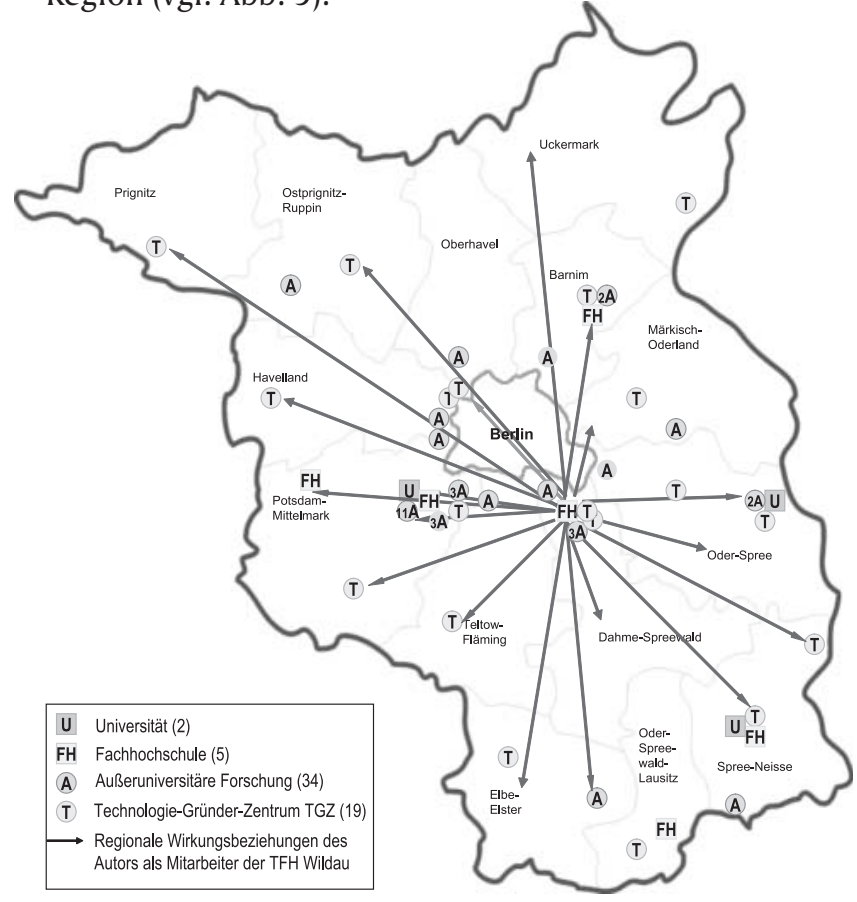

Abb. 9: Räumliche Wirkungsorientierung der Forschungsgruppe „Regionale Innovationscluster" der TFH Wildau
Würde man dazu die Ergebnisse weiterer Professorenkollegen aus ingenieurtechnischen und anderen wirtschaftswissenschaftlichen Disziplinen hinzufuigen, würde ein so dichtes Netz abzubilden sein, dass es hier nicht mehr sinnvoll darstellbar wäre. Allein die Professoren des Fachbereichs Betriebswirtschaft/Wirtschaftsinformatik der TFH Wildau unterhalten zu ca. 70 Unternehmen unterschiedlichste Kontakte, von denen etwa drei Viertel in der Region Berlin-Brandenburg angesiedelt sind.

Die Fachhochschule konstituiert gemeinsam mit anderen regionalen Akteuren unterschiedlich dimensionierte projektdefinierte Regionen bzw. ist daran beteiligt. Das betrifft beispielsweise über ein InnoRegio-Projekt eine „Biotechnologieregion“ im Raum Wildau - Potsdam - Luckenwalde oder über eine regionale Entwicklungspartnerschaft „EQUAL“ eine „Region“ Guben - Herzberg - Belzig - Wildau - Oranienburg - Brandenburg - Neuruppin. Durch andere Projekte werden mehrere Landkreise erfasst. Die in Ausprägung befindliche „Luft- und Raumfahrt-Region Berlin-Brandenburg“" umfasst etwa Berlin, Schönefeld, Wildau, Ludwigsfelde, Dahlewitz und Cottbus. In einem vom Autor initiierten und geleiteten „Forschungsverbund Regionale Innovationssysteme Berlin-Brandenburg" konstituiert sich zunehmend eine „Region“ Wildau, Potsdam, Teltow, Brandenburg, Berlin, Neuenhagen, Eberswalde, Frankfurt/Oder, auch durch zunehmende Einbeziehung der regionalen Technologie- und Gründerzentren (TGZ), Kammern, Verbände und Verwaltungen der Landkreise in Aktivitäten des Verbundes (vgl. Voß, 2002).

\section{Regionale Rahmenbedingungen für die Wirksamkeit Brandenburger Hochschulen}

Die regionale Wirksamkeit einer Hochschule muss sicher auch auf dem Hintergrund der regionalen Hochschullandschaft und deren Leistungsfähigkeit sowie Wettbewerbsfähigkeit im Vergleich zu anderen Regionen analysiert und vor allem bewertet werden. Die Bezugsebene hier bildet sinnvollerweise das Bundesland.

In der Übersicht 4 sind einige ausgewählte Indikatoren für die Charakterisierung und den Vergleich solcher Rahmenbedingungen angefuihrt, die durchaus unterschiedlich hinsichtlich ihrer fördernden oder hemmenden Einflüsse auf die regionale Wirksamkeit einer Brandenburger Hochschule zu diskutieren sind (vgl. Übersicht 4).

Zunächst fällt auf, dass das Land Brandenburg bei $50 \%$ der 14 ausgewählten Indikatoren den letzten und bei ca. weiteren 30 \% den vorletzten Rangplatz unter den fünf neuen Bundesländern einnimmt. Vergleicht man Brandenburg, in dessen Zentrum die Hauptstadt Berlin liegt, mit dem Freistaat Sachsen (das sollte die für die Hauptstadtregion angemessene Herausforderung für die Zukunft sein), dann erreicht das Land in den meisten Kennziffern nur das Niveau von 20 bis 50 \% bezogen auf Sachsen.

Beide Betrachtungen verweisen auf nicht gerade vorteilhafte Rahmenbedingungen für die Wettbewerbsfä- 


\begin{tabular}{|c|c|c|c|c|c|}
\hline Indikator & Maßeinheit & $\begin{array}{l}\text { Zeitpunkt/ } \\
\text { Zeitraum }\end{array}$ & Brandenburg & Vergleiche & $\begin{array}{l}\text { Rangplatz } \\
\text { Bbg. (NBL) }\end{array}$ \\
\hline Hochschulen & Zahl je 1 Mio. Einw. & 1999 & 4,2 & $\begin{array}{l}5,3 \mathrm{NBL} \\
5,8 \text { Sachsen }\end{array}$ & 5. \\
\hline Hochschulexterne Forschungseinrichtungen & Zahl je 1 Mio. Einw. & 2001 & 11,2 & $\begin{array}{l}7,8 \mathrm{NBL} \\
7,2 \text { Sachsen }\end{array}$ & 1. \\
\hline Ausgaben der Hochschulen & DM/Einw. & 1999 & 258 & $\begin{array}{l}35 \% \text { zu D gesamt } \\
40 \% \text { zu Sachsen }\end{array}$ & 5. \\
\hline Hochschulabsolventen & Zahl je 100 Tsd. Einw. & 2002 & 178 & $\begin{array}{l}47.5 \% \text { zu D gesamt } \\
48,9 \% \text { zu Sachsen }\end{array}$ & 5. \\
\hline $\begin{array}{l}\text { Hauptberufliches Personal der Hochschulen } \\
\text { für FuE }\end{array}$ & $\begin{array}{l}\text { Vollzeitäquiv. } \\
\text { je } 100 \text { Tsd. Einw. }\end{array}$ & 1998 & 53 & $\begin{array}{l}48 \% \text { zu NBL } \\
35 \% \text { zu Sachsen }\end{array}$ & 5. \\
\hline Professoren in Hochschulen & $\begin{array}{l}\text { Zahl } \\
\text { Zahl/1000 Studenten }\end{array}$ & $\begin{array}{l}1999 \\
1998\end{array}$ & $\begin{array}{l}722 \\
26\end{array}$ & $\begin{array}{l}32 \% \text { zu Sachsen } \\
88 \% \text { zu Sachsen }\end{array}$ & $\begin{array}{l}5 . \\
5 .\end{array}$ \\
\hline Einnahmen der Hochschulen aus Drittmitteln & $\begin{array}{l}\text { Mio. DM } \\
\text { Veränderung \% }\end{array}$ & $\begin{array}{l}1999 \\
1995-99\end{array}$ & $\begin{array}{l}59 \\
100 \\
\end{array}$ & $\begin{array}{l}21 \% \text { zu Sachsen } \\
\text { Sachsen: } 20 \%\end{array}$ & $\begin{array}{l}4 . \\
1 .\end{array}$ \\
\hline $\begin{array}{l}\text { Drittmitteleinnahmen zu Gesamtausgaben- } \\
\text { hochschule }\end{array}$ & $\begin{array}{l}\text { Ausgaben } 100 \% \\
\text { Veränderung \% }\end{array}$ & $\begin{array}{l}1999 \\
1995-99\end{array}$ & $\begin{array}{l}8,8 \\
76\end{array}$ & $\begin{array}{l}\text { Sachsen: } 9,3 \% \\
\text { Sachsen: } 11,6 \%\end{array}$ & $\begin{array}{l}2 . \\
1 .\end{array}$ \\
\hline Drittmittel aus der gewerblichen Wirtschaft & Mio. DM & 1999 & 15 & $22,5 \%$ zu Sachsen & 4. \\
\hline FuE Personal im Wirtschaftsektor & Zahl & 1997 & 2860 & $25 \%$ zu Sachsen & 4. \\
\hline Interne FuE Aufwendungen der Privatwirtschaft & DM/Einw. & 1997 & 178 & $55,6 \%$ zu Sachsen & 3. \\
\hline Kooperationen der Betriebe mit Hochschulen & $\%$ koop. Betriebe & 1998 & 47,4 & Sachsen: 53,7\% & 5. \\
\hline Patentanmeldungen & Zahl je 100 Tsd. Einw. & 1995-99 & 64 & $58 \%$ zu Sachsen & 4. \\
\hline
\end{tabular}

Übersicht 4: Rahmenbedingungen für die regionale Wirksamkeit von Hochschulen im Land Brandenburg nach ausgewählten Indikatoren (Nach: Franz u.a. 2001; Rosenfeld/Heimpold 2001, IWD 2004; eigene Zusammenstellung und z. T. Berechnungen)

higkeit Brandenburger Hochschulen hinsichtlich ihrer regionalen Wirksamkeit im Vergleich zu Hochschulen anderer Bundesländer. Die Indikatoren müssen unter diesem speziellen Fokus aber von verschiedenen Seiten betrachtet werden. Einerseits bedeuten sicher für Sachsen z. B. die größere Hochschuldichte, die dreifache Zahl von Professoren, die doppelte Zahl an Hochschulabsolventen, der hohe Anteil von hauptberuflichem Potenzial der Hochschulen für FuE und die 60 \% mehr Ausgaben des Landes für die Hochschulen zunächst eine bessere Ausgangsbasis, mehr Potenzial und damit auch Möglichkeiten entsprechend stärkere Wirksamkeit im gesamten Spektrum zu entwickeln. Das Land Brandenburg hat hier in vieler Hinsicht weiteren Aufholbedarf. Andererseits bleibt immer auch die entscheidende Frage, was Hochschulen unter mehr oder weniger guten derartigen Rahmenbedingungen dann tatsächlich daraus machen, wie sie damit umgehen und darauf reagieren.

Die Technische Fachhochschule Wildau beispielsweise reagiert darauf mit einer Ausdehnung ihrer regionalen Aktivitäten auf das gesamte Bundesland Brandenburg. Sie wird auch zunehmend von unterschiedlichen Akteuren als so agierend wahrgenommen, in regionale Projekte in allen Landesteilen einbezogen und angefragt. Die Tatsache, dass die TFHW ein Drittel zusätzlich zur Haushaltssumme über Drittmittel einwirbt und sich die Einnahmen der Brandenburger Hochschulen aus Drittmitteln von 1995 - 1999 um $100 \%$ erhöht haben, stimmen zumindest optimistisch, ebenso wie der starke regionale Besatz mit hochschulexternen Forschungseinrichtungen (Institute der Leibnitzgesellschaft, Max-Planck-Gesellschaft, Fraunhofer Gesellschaft und der Ressortforschung). In stärkerer Kooperation mit letzteren und in dem Engagement der Brandenburger Wirtschaft für die eigene Forschung und damit auch die Kooperation mit den Hochschulen liegen offensichtlich noch erhebliche Reserven (vgl. Übersicht 4).

\section{Fazit}

Wie das vorgestellte Konzept zur strukturierten Analyse und Bewertung der regionalen Wirksamkeit von Hochschulen und die unterlegten Beispiele der Technischen Fachhochschule Wildau zeigen, bestehen ausgehend von den prinzipiellen Funktionen einer Hochschule und in Abhängigkeit von der Nachfrage sowie den Voraussetzungen einer Region eine große Vielzahl und Vielfalt von Spezialisierungs- und Differenzierungsmöglichkeiten.

Die regionale Wirksamkeit einer Hochschule beschränkt sich weder auf bildungsseitige noch auf raumwirtschaftliche Effekte und lässt sich auch nicht in einer Gesamtkennziffer synthetisieren oder etwa gar monetär ausdrüicken.

Das tatsächlich erreichte Spektrum und die Intensität förderlicher Wirkungen einer Hochschule auf regionale Zielbildungs- und Gestaltungsprozesse in solchen Bereichen wie Bildung, Wissenschaft, Wirtschaft, Verwaltung und Politik im Spannungsfeld der dargestellten Wirkungsräume hängt in erster Linie von der Kompetenz, der Motivation und dem Engagement der Professoren ab. Wenn diese eine offensive Haltung gegenüber der Region und ihren Problemen einnehmen und nicht nur darauf warten, dass Unternehmen, Verbände und Verwaltungen mit ihren Problemen auf die Hochschule zukommen, sondern durch enge persönliche Kontakte zu regionalen Akteuren, durch Mitarbeit in Gremien, Verbänden, Vereinen und Netzwerken diese Probleme und Erfordernisse selbst gut kennen, lassen sich auch die Leistungspotenziale der Hochschule effizient und effektiv für die Region erschließen und weiterentwickeln.

Eine solche Arbeitsweise von Professoren setzt natuirlich gleichermaßen hohe Aufgeschlossenheit und entsprechendes Engagement seitens der jeweiligen Hochschul- 
leitung voraus und muss durch eine große Einsatzbereitschaft der Verwaltung sowie dienstleistender Bereiche der Hochschule zur Unterstuitzung regionalorientierter Projekte der Professoren und ihrer Mitarbeiter flankiert werden. Insgesamt muss an der Hochschule auch ein ausgeprägtes Klima für eine starke regionale Wirksamkeit entwickelt werden. Dies wird auch unterstuitzt durch entsprechende Anreizsysteme, wie etwa in der Technischen Fachhochschule Wildau durch Auslobung eines Lehrpreises bzw. eines Forschungspreises, sowie durch besondere Berüicksichtigung von Drittmittelaktivitäten bei der leistungsbezogenen Aufteilung von Haushaltsmitteln der Fachhochschule auf die Fachbereiche.

Auf Seiten regionaler Akteure, der einzelnen Unternehmen, aber auch ihrer Verbände, der Kammern, Regionalverwaltungen und regionalen Entwicklungsinitiativen ist ebenfalls eine hohe Aufgeschlossenheit und ein aktives Zugehen auf die Hochschule und ein offensives Einbeziehen der Kompetenzen der Hochschulmitarbeiter und der Studenten in die Lösung regionaler Entwicklungsprobleme erforderlich.

Schließlich wirken auch bestimmte Maßnahmen auf der Ebene der Länder und des Bundes fördernd auf die regionale Wirksamkeit von Hochschulen. Beispielsweise hat das Bundesministerium für Bildung und Forschung sein von 1992 bis 2003 aufgelegtes Förderprogramm „Anwendungsorientierte Forschung und Entwicklung an Fachhochschulen“ weiter profiliert und seit 2004 unter dem Titel „FH $\mathrm{FH}^{3}$ - Angewandte Forschung und Entwicklung für die Wirtschaft" neu orientiert. Jetzt werden für die zu beantragenden Projekte nicht nur die Einbeziehung von Unternehmen, sondern zusätzlich Kooperationen mit anderen Fachhochschulen und Forschungseinrichtungen, Verbänden u. ä. gefordert. Dies unterstuitzt in erster Linie auch die regionale Wirksamkeit, weil in der Regel solche jetzt größeren Verbünde auf regionaler Ebene zustande kommen. Damit wird nicht nur die Drittmittelfähigkeit der Fachhochschulen gefördert, sondern auch ihre Integration in regionale Netzwerke.

Hervorzuheben ist auch, dass das Ministerium für Wissenschaft und Kultur des Landes Brandenburg mit Beginn des Haushaltsjahres 2004 ein neues leistungsbezogenes Modell zur Haushaltsbildung für die Hochschulen anwendet. In diesem Modell werden ebenfalls Kriterien und Kennziffern erstmalig bzw. verstärkt zur Anwendung gebracht, die z. B. Drittmittelaktivitäten in der Forschung und im Technologietransfer erfassen.

Beide beispielhaft genannten Maßnahmen werden sich zukünftig fördernd auf den Wettbewerb der Hochschulen hinsichtlich ihrer regionalen Wirksamkeit auswirken. Es ist zu erwarten, dass sich die Tendenz verstärken wird, dass Hochschulen zu einem Nukleus von Regionalen Innovationssystemen werden, wenn sie die oben diskutierten regionalen Wirkungsräume nachfragegerecht zunehmend auf höherem Niveau ausfuillen können und auf diesem Wege zu Schlïsselakteuren in ihren Regionen werden (vgl. Voß, 2002).

\section{Anmerkungen}

[1] Ausfuihrliche Quellenangaben siehe Literaturverzeichnis

[2] Dabei wird wesentlich auf statistische Angaben aus einer aktuellen Präsentation „Technische Fachhochschule Wildau - Fakten, Zahlen, Entwicklungen" des Präsidenten der TFH Wildau, Professor Dr. László Ungvári, zurückgegriffen

[3] Der Rest der Befragten machte in einer schriftlichen Befragung von Absolventen der TFH Wildau im Jahr 2002 keine Angaben.

\section{Literatur}

Bauer, E.-M.: Die Hochschule als Wirtschaftsfaktor. Eine systemorientierte und empirische Analyse universitätsbedingter Beschäftigungs-, Einkommens- und Informationseffekte - dargestellt am Beispiel der Ludwig-Maximilians-Universität München, Kallmüinz / Regensburg 1997 (Münchner Studien zur Sozial- und Wirtschaftsgeographie, Bd. 41).

Benson, L.: Regionalwirtschaftliche Effekte der Universität Trier. Eine Untersuchung der Auswirkungen auf die regionale Wirtschaft während der Leistungserstellungsphase, Trier 1997.

Blume, L.; FROMM, O.: Regionalökonomische Bedeutung von Hochschulen. Eine empirische Untersuchung am Beispiel der Universität Gesamthochschule Kassel, Wiesbaden 2000.

Drude, M.: Die Albert-Ludwigs-Universität Freiburg als Wirtschaftsfaktor für die Region, Freiburg/Br. 1995.

Ewers, H.-J.; WETTMANN, R.W.: Innovationsrückstände und regionale Innovationspolitik im ländlichen Raum. In: Innovationsförderung im ländlichen Raum; Informationen zur Raumentwicklung, Heft 7/8, 1980.

Stifterverband: FuE Info 2/2003; hrsg. von Wissenschaftstatistik gGmbH im Stifterverband fuir die deutsche Wirtschaft, Essen 2003

Franz, P.; Rosenfeld, M. T. W.; Roth, D.: Was bringt die Wissenschaft für die Wirtschaft in einer Region? Empirische Ergebnisse zu den Nachfrageeffekten und Hypothesen über mögliche Angebotseffekte der Wissenschaftseinrichtung in der Region Halle, Institut für Wirtschaftsforschung Halle (IWH) Juni 2002 Diskussionspapiere Nr. 163.

Ganser, K.: Der Beitrag der Hochschulen zur Innovationsförderung im ländlichen Raum. In: Innovationsförderung im ländlichen Raum; Informationen zur Raumentwicklung, Heft 7/8, 1980.

Geppert, K.; Vesper, D.: Zur regionalwirtschaftlichen Bedeutung der Berliner Hochschulen, Deutsches Institut für Wirtschaftsforschung, Berlin 1997.

GEW (Hrsg.): Fachhochschulen in der deutschen Hochschullandschaft. Wo stehen sie? Wo wollen sie hin? Standpunkt der GEW, 1996. (www.gew-thueringen.de)

Giese, E.; Aberle, G.; Kaufmann, L.: Wechselwirkungen zwischen Hochschule und Hochschulregion - Fallstudie Justus-Liebig-Universität Gießen, 2 Bde., Gießen 1982.

Gloede, K.; Schirmag, T.; Schöler, K.: Ökonomische Wirkungen der Universität Potsdam auf die Region, Frankfurt/M. u. a. 1999.

Große, U.; Hartmann, F; Voß, R.: Internationale Trends in der Biotechnologie. Auswertung aktueller Trends in biotechnologischen Innovationslinien mit Relevanz für kleine und mittlere Unternehmen, Berlin 1998. 
Hecht, M.: Innovationspotentiale in der Region. Die regionalwirtschaftliche Bedeutung der Universität Greifswald, Regensburg 1998.

Heimpold, G.: Die Unternehmenslïcke in Ostdeutschland schließt sich noch nicht. Wirtschaft im Wandel 12/2003, S. 330.

Holtkamp, R.: Forschung an Fachhochschulen. Ergebnisse einer empirischen Untersuchung. HIS-Kurzinformationen A2/1995.

IWD: IWD, Nr. 13, 25. März 2004, S. 3.

Kleinhenz, G.: Die Universität - Auswirkungen auf die Stadt Passau und das Umland, Passau 1990.

Knödler R.; Tivig, T.: Die Universität Rostock als regionaler Wirtschaftsfaktor, Rostock 1996 (Thünen-Series of Applied Economic Theory, Working Paper No. 3).

Koschatzky, K.; Zenker, A.: Innovative Regionen in Ostdeutschland - Merkmale, Defizite, Potentiale. Arbeitspapier Regionalforschung Nr. 17, April 1999, Institut für Systemtechnik und Innovationsforschung, Karlsruhe.

Kuhn, R.: Perspektiven und Probleme der Siedlungsentwicklung in den industriegeprägten Räumen der Peripherie Brandenburgs am Beispiel des brandenburgischen Teils der Lausitz. In: Zöpel, Ch. (Hrsg.): Brandenburg 2025 in der Mitte Europas, Band 2, Berlin/Potsdam 2002, S. 443-451.

Möller, J.; Oberhofer, W.: Universität und Region, Regensburg 1997.

Niermann, U.: Wirtschaftsfaktor Universität - Eine inputoutput-orientierte Analyse am Beispiel der Universität Bielefeld, 2 Bde., Münster 1996.

Oser, U.; Schröder, E.: Die Universität Konstanz als Wirtschaftsfaktor für die Region, Konstanz 1995 (Center for International Labor Economics).

Pfähler, W.; Clermont, C.; Gabriel, C.; Hofmann, U.: Bildung und Wissenschaft als Wirtschafts- und Standortfaktor - Die regionalwirtschaftliche Bedeutung der Hamburger Hochschulbildungs- und Wissenschaftseinrichtungen, Hamburg 1997.

Pfaff, M.; Becker, W.: Universität und Wirtschaftsstruktur. Zur kommunal- und regionalwirtschaftlichen Bedeutung der Universität Augsburg, Augsburg 1990.

Rosenfeld, M. T. W.; Heimpold, G.: Öffentliche Forschung in Ostdeutschland: ein Hoffnungsträger für den wirtschaftlichen Aufschwung. Wirtschaft im Wandel 15/2001, S. 393-404.

Schultz, A.: Die Universität als Wirtschaftsfaktor. Eine Untersuchung der regionalwirtschaftlichen Einkommens- und Beschäftigungseffekte sowie des Wissenstransfers der Martin-Luther-Universität Halle-Wittenberg, Halle/S. 2001 (Diplomarbeit).

Voigt, E.: Die Universität als Wirtschaftsfaktor am Beispiel der TU Ilmenau. In: Raumforschung und Raumordnung, 54. Jg., H. 4, 1996, S. 283-289.

Voss, R.: Regionale Innovationssysteme als Gegenstand eines neuen Forschungsverbundes der Region Berlin-Brandenburg. In: Voß, R. (Hrsg.): Regionale Innovationssysteme, Berlin 2002 (Wildauer Schriftenreihe Innovationsmanagement; Bd. 2), S. 25-46.

Willauschus, A.: Wirtschaftliche Verflechtungen zwischen Hochschulen und Stadt. Eine empirische Analyse am Beispiel der Stadt Münster und ihren Hochschulen, Baden-Baden 1979.

Wissenschaftsrat (Hrsg.): Empfehlungen zur Entwicklung des Wissenschaftsrates zur Entwicklung der Fachhochschulen in den 90er Jahren, Köln 1991

\section{Autor}

Prof. Dr. phil. Rainer Voß

Technische Fachhochschule Wildau

Dekan des Fachbereiches BW/WI

Leiter der Forschungsgruppe „Regionale Innovationscluster"

Tel. +493375 508-951

E-Mail: rvoss@igw.tfh-wildau.de 